\title{
PAUTAS TEÓRICAS Y METODOLÓGICAS PARA DE- TERMINAR LA PRODUCTIVIDAD DE LOS PREDI- CADOS AFIJALES EN UNA BASE DE DATOS LÉXICA DEL INGLÉS ANTIGUO ${ }^{1}$
}

\author{
Elisa González Torres y Ana Ibáñez Moreno²
}

\begin{abstract}
Resumen: Este artículo establece las bases teóricas y metodológicas para el estudio de la productividad de los predicados afijales en una base de datos del inglés antiguo. Tras un breve análisis del estado de la cuestión en productividad léxica, se propone distinguir la productividad cualitativa de la cuantitativa. La productividad cualitativa se analiza desde el punto de vista de la distribución y el comportamiento de los predicados afijales. De manera tentativa, los predicados afijales $a-, a e-, b e-$, for-, ofer- y to- se consideran cuantitativamente productivos. También se propone y se ilustra la diferencia entre el fenómeno de hapax legomena absoluto y relativo. Tras un análisis de unos mil trescientos predicados verbales derivados, los predicados afijales for-, on- y to- se confirman como productivos.
\end{abstract}

Palabras clave: Inglés antiguo, gramática, morfología, derivación, afijos.

\begin{abstract}
This journal article establishes the theoretical and methodological bases for the study of the productivity of affixal predicates in a database of Old English. After revising critically the state of the art about lexical productivity, we propose to distinguish between qualitative and quantitative productivity. Qualitative productivity, on the one hand, is analised from the point of view of distribution and the behaviour of affixal predicates. In a tentative way, the affixal predicates $a_{-}, a_{-}$, , be-, for-, ofer-y to- are considered quantitatively productive. Besides, it is proposed and illustrated the difference between the hapax legomena phenomenon absolute and relative. After an analysis of about one thousand three hundred derived verbal predicates, the affixal predicates for-, on- and to- qualify as productive.
\end{abstract}

Keywords: Old English, grammar, morphology, derivation, affixes.

\section{INTRODUCCIÓN}

Este artículo establece pautas teóricas y metodológicas para el estudio de la productividad del léxico del inglés antiguo. Se propone distinguir productividad cuantitativa y

\footnotetext{
1 Fecha de recepción: abril 2007.

Fecha de aceptación y version final: julio 2007.

La investigación que se recoge en este trabajo ha sido financiada con cargo al proyecto ANGI03/08 (CAR). Una versión preliminar de este trabajo se presentó en el XXII Congreso Internacional de AESLA.

2 Elisa González Torres es Becaria de Investigación, Departamento de Filologías Modernas, Universidad de la Rioja; $\bowtie$ elisa.gonzalez@unirioja.es. Ana Ibáñez Moreno es Profesora Ayudante, Departamento de Filologías Modernas, Universidad de la Rioja; $\bowtie$ ana.ibanezm@unirioja.es.
} 
cualitativa. Dentro de la productividad cuantitativa, se presentan casos del fenómeno de hapax legomena absolutos y relativos. La productividad relativa se estudia desde la doble perspectiva de la distribución y el comportamiento de los predicados afijales del precampo verbal (prefijos). Para determinar la productividad, se busca la coincidencia de los análisis cuantitativos y cualitativos, tanto de distribución como de comportamiento. El análisis se ha llevado a cabo a partir de unos mil trescientos predicados verbales derivados, correspondientes a un vaciado de la letra A de An Anglo-Saxon Dictionary (Bosworth y Toller 1973).

\section{EL CARÁCTER ASOCIATIVO DEL LÉXICO ANGLOSAJÓN}

Numerosos autores han insistido tanto en el carácter asociativo del léxico anglosajón (Romano 2001), que recurre a la composición y derivación más que al préstamo de nuevos términos. Efectivamente, y empezando por la composición, se ofrece en (1) una ilustración de la productividad de este fenómeno por medio de algunos compuestos de cyric "church":

(1)

cyric-awe "ecclesiastical marriage", cyric-belle "church bell", cyric-boc "church book", cyric-bot "church repair", cyric-bryce "church breach", cyric-burh "church city", cyric-dor "church door", etc.

También la derivación, de la que se ofrece una ilustración en (2) por medio de la afijación al predicado verbal settan "set", se manifiesta en inglés antiguo como un proceso productivo con posibilidad de contribuir de manera muy destacada a la creación léxica en la lengua:

(2)

asettan "place", besettan "appoint", forsettan "obstruct", foresettan "place before", gesettan "people, garrison", ofsettan "afflict", onsettan "oppress, tosettan "dispose", unsettan "put down", widsettan "resist".

Sin embargo, estas afirmaciones de carácter general deben ser matizadas. En lo que se refiere al carácter asociativo del léxico, suele contraponerse el léxico del inglés antiguo, al que se considera asociativo en tanto que eminentemente germánico y con tendencia a la creación léxica por medio de composición y derivación, al léxico del inglés medio y periodos posteriores, al que se tiene por disociativo, dado que da cabida a palabras germánicas y románicas y hace uso generalizado del préstamo léxico. Si repasamos los prestamos latinos ya en el léxico del inglés antiguo, o recordamos los numerosos prefijos y sufijos del inglés antiguo que siguen presentes en la lengua, probablemente llegaremos a la conclusión de que existen tendencias más acusadas a la creación léxica o al préstamo léxico en distintos periodos históricos, más que la sustitución de un procedimiento de creación léxica por otro de préstamo.

En cuanto a la productividad, Baugh y Cable (1987: 182) y Romano (2001: 387), entre otros, ponen de manifiesto que la posibilidad de utilizar de manera productiva los afijos del inglés antiguo desciende conforme avanza el periodo. Kastovsky (1992: 377) data el 
declive de la productividad afijal anglosajona en el final del siglo X. Asumiendo que, en efecto, la productividad afijal descendió, estas afirmaciones deberán respaldarse con datos empíricos cualitativos y, si es posible, cuantitativos. ${ }^{3}$

Este breve estado de la cuestión no hace sino resaltar algunas líneas de investigación pendientes en el campo del léxico del inglés antiguo, entre las que destaca el carácter productivo de la creación léxica en inglés antiguo, bien sea ésta el producto de la derivación o de la composición. Como afirma Kastovsky (1992: 360), hace falta aún mucho trabajo hasta llegar a una descripción de la derivación léxica en inglés antiguo que dé cuenta de los aspectos transparentes y opacos de dicha derivación, a la vez que mantenga el equilibrio necesario entre el estudio sincrónico y el diacrónico.

\section{APROXIMACIÓN A LA PRODUCTIVIDAD DEL LÉXICO ANGLOSAJÓN}

De acuerdo con lo expuesto, a continuación nos ocupamos de la cuestión de la productividad del léxico anglosajón a partir de un vaciado de los predicados verbales primitivos y derivados de la letra A de An Anglo-Saxon Dictionary (Bosworth y Toller 1973). No está fuera de lugar en este punto hacer una aclaración terminológica. La creación léxica está constreñida. Esto es, no es posible combinar cualquier predicado no afijal con cualquier predicado afijal en procesos derivativos ( $\mathrm{y}$, problamente, tampoco es posible combinar cualquier predicado no afijal con cualquier predicado no afijal en procesos compositivos). Por tanto, es preciso determinar cuáles y de qué tipo son las restricciones a la derivación, en virtud de las cuales sólo algunas de las combinaciones sistemáticas de predicado afijal y base tienen aceptación en la norma. Dichas restricciones, basándonos en Dik (1986) y en Plag (1999), se pueden dividir en formales y funcionales. Las restricciones formales a la derivación serán de tipo fonológico (es decir, la interrelación de la forma fonológica de la base y del predicado afijal) y de tipo morfológico (como, por ejemplo, el origen germánico o romance de los predicados afijales y las bases). Las restricciones funcionales a la derivación serán de tipo semántico (derivadas de los papeles semánticos o consideraciones de iconicidad) o cognitivo (como la maximización y la economía).

De lo dicho se debe concluir que la productividad debe relacionar la creación léxica sistemática (no restringida por el sistema) y la creación léxica aceptada (incluida en la norma). En el eje diacrónico, sin embargo, se plantean dificultades adicionales a la hora de definir y medir la productividad léxica. Katamba (1993: 67) se refiere expresamente al hecho de que la productividad está sujeta a variación diacrónica:

Productivity is subject to the dimension of time. A process which is very general during one historical period may become less so at a subsequent period. Conversely, a new process entering a language may initially affect a tiny fraction of eligible inputs before eventually applying more widely.

\footnotetext{
Ver Hiltunen (1983), aunque este autor se limita a los prefijos no separables $a-$, be-, for-, ge-, of-, on- y to-. Ver también Lass (1975: 235-241).
} 
De esta cita se sigue la necesidad de medir la productividad de los predicados afijales con referencia a periodos determinados de tiempo, sincronías, y compararlos entre sí en la diacronía. No se dice nada, sin embargo, de las lenguas que ya no se hablan pero de las que queda suficiente evidencia textual para llevar a cabo un estudio lingüístico completo y riguroso, como es el caso del inglés antiguo. ¿Es la productividad léxica una cuestión relevante? Entendemos que sí, desde el momento en que ni todos los predicados afijales ni todos los predicados no afijales muestran las mismas propiedades de codificación ni de comportamiento, bien sea intra-clase o inter-clase. Concluimos esta parte con una definición de productividad aplicable al estudio del léxico anglosajón: la productividad es la ratio entre la derivación léxica posible y la derivación léxica atestiguada.

La siguiente cuestión es cómo se mide la productividad. Bauer (1983: 63) define la productividad como rule-governed innovation y la caracteriza como absoluta: los hablantes pueden o no pueden producir y comprender nuevas palabras resultado de procesos de derivación léxica. Así, este autor distingue la productividad de la semiproductividad, que se produce cuando los predicados afijales susceptibles de combinarse con una base no se distribuyen libremente (así, certainty es sistemático y acorde a la norma pero certainnes es sistemático pero no acorde a la norma).

Beard (1998: 62) relaciona la productividad con la transparencia, la cual se debe a la ausencia de alomorfismo. Cuando existe alomorfismo, la combinación de predicado afijal y base es más opaca y menos probable de que tenga lugar. Este mismo autor vincula el isomorfismo y la productividad. Así, los derivados agentivos en -er como baker son mucho más frecuentes que los derivados por morfema cero, por tanto sin marca expresa, como "cook" -"cook" .

Los autores citados anteriormente comparten una visión absoluta de la productividad con otros como Spencer (1991) o Stump (2001). Seguiremos, más bien, a lingüistas como Katamba (1993) o Aronoff y Anshen (1998), quienes entienden la productividad de manera relativa, es decir, como una gradación entre la falta absoluta de restricciones a la derivación y la imposibilidad total de derivar nuevos predicados. Más aún, Aronoff y Anshen distinguen aspectos cualitativos y cuantitativos de la productividad; y tanto Katamba como Arnoff y Anshen añaden la variable de la frecuencia de la base a la variable más usual en la literatura de la frecuencia del predicado afijal.

\section{PRODUCTIVIDAD CUANTITATIVA}

De la discusión teórica y terminológica anterior se puede concluir que se debe determinar la productividad léxica como una gradación y de manera más textual, no tan orientada a la creación de predicados derivados (Bauer 1983: 99) como a la existencia de predicados derivados. Es, por tanto, un objetivo central de esta investigación determinar el inventario de predicados primitivos y derivados, teniendo en cuenta que debe establecerse con referencia a los predicados derivados existentes, no a los predicados susceptibles de ser derivados. Esto equivale a decir que los inventarios de predicados primitivos y derivados serán el resultado de la aplicación de pruebas indirectas basadas en la distribución y la descomposición que deberán determinar si una secuencia identificable a priori como un predicado afijal constituye o no tal predicado afijal. Por distribución y descomposición, el vaciado de la letra A 
de An Anglo-Saxon Dictionary (Bosworth y Toller 1973) arroja el siguiente inventario de predicados afijales de precampo verbal, que se presentan con sus variantes fonológicas y ortográficas y con distinción de la cantidad vocálica:

(3)

æ/æ:, æd/ed, af/æf/æ:f/of, age:an/onge:an/a:ge:n, an/æn/æ:n/en/and/ond, a:r/æ:r/ær, æt/a:t, after, be/bi/bi:, for/fore/forg, for $\partial$, in, mis, ofer, on, onemn, o $\partial$, to/to:, un, under,

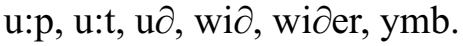

A continuación se muestra la productividad absoluta de los predicados afijales identificados, siempre basándonos en los datos aportados por el vaciado de la letra A. Las formas prototípicas se muestran en negrita, mientras que el resto son las diferentes variantes ortográficas de dichas formas:

(4)

\begin{tabular}{|l|l|l|l|l|l|l|l|l|l|l|l|l|l|l|}
\hline a: & æ, & $\begin{array}{l}\text { æd, } \\
\text { ed }\end{array}$ & $\begin{array}{l}\text { af, } \\
\text { æf, } \\
\text { a:f, } \\
\text { of }\end{array}$ & $\begin{array}{l}\text { an, } \\
\text { æn, } \\
\text { a:n, } \\
\text { and, } \\
\text { ond }\end{array}$ & $\begin{array}{l}\text { a:r, } \\
\text { æ:r, } \\
\text { ær }\end{array}$ & $\begin{array}{l}\text { at, } \\
\text { a:t }\end{array}$ & after & $\begin{array}{l}\text { be, } \\
\text { bi, } \\
\text { bi: }\end{array}$ & $\begin{array}{l}\text { for, } \\
\text { fore, } \\
\text { forg }\end{array}$ & for & in & mis & ofer & on \\
\hline 882 & 42 & 23 & 100 & 67 & 5 & 119 & 12 & 317 & 216 & 35 & 53 & 45 & 121 & 202 \\
\hline
\end{tabular}

\begin{tabular}{|c|c|c|c|c|c|c|c|c|c|c|c|}
\hline onemn & $\begin{array}{l}\text { a } g \text { e : a } n, \\
\text { o } n \text { g e a } n, \\
\text { a:ge:n }\end{array}$ & $\mid \begin{array}{ll}t & 0 \\
\text { to: }\end{array}$ & $\mathrm{o} \partial$ & un & under & $\mathrm{u}: \mathrm{p}$ & U:t & $\mathrm{u} \partial$ & wid & wider & ymb \\
\hline 1 & 10 & 152 & 53 & 39 & 57 & 26 & 24 & 1 & 44 & 11 & 67 \\
\hline
\end{tabular}

Un primer comentario respecto a (4) es que en esta comunicación nos ocupamos de la productividad de los predicados, no en la productividad de los procesos. Los datos cuantitativos de (4), en todo caso, evidencian que hay dos predicados afijales que representan el grado mínimo de productividad, denominado hapax legomena (Lieber 1992: 4): predicados afijales que sólo se combinan con un predicado no afijal. Así, como se ilustra en (5.a), onemn "alongside" forma parte de una cadena derivativa con el predicado verbal Drowian, "endure" para producir onemndrowigan "sympathize". Onemn "alongside" es un hapax legomena absoluto. No hay ningún otro derivado de ninguna categoría léxica con este predicado afijal. El caso de $u \partial$ - en (5.b) es algo distinto. Este predicado afijal forma parte de una cadena derivativa con witan "know" para producir $u \partial$ witian "study philosophy". Desde la perspectiva de los predicados verbales, este predicado afijal constituye un hapax legomena porque no hay más predicados verbales derivados de los que forme parte. Sin embargo, a diferencia de onemn-, $u \partial$ - forma parte de cadenas derivativas que producen derivados no verbales, dados en (5.c). Por tanto, $u \partial$ - debe considerarse un hapax legomena relativo. 
(5)

a. Drowian "endure": onemn-drowigan "sympathize"

b. witan "know": $u \partial$-witian "study philosophy"

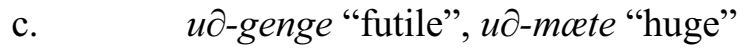

Los datos cuantitativos de (4) también ponen de manifiesto que los predicados afijales de precampo verbal cuantitativamente más productivos son $a^{-}$, cet-, be-, for-, ofer-, on-, y to-. Aunque $a$ - destaca respecto al resto, hay que tomar este dato con prudencia puesto que el hecho de que el vaciado llevado a cabo sea el de la letra A introduce muy probablemente un sesgo en la cuantificación. La investigación ulterior solventará esta cuestión.

\section{PRODUCTIVIDAD CUALITATIVA}

Cualitativamente, proponemos atender a la distribución y al comportamiento de los predicados afijales para medir la productividad. En lo que concierne a la distribución, sostenemos que es más productivo un predicado afijal capaz de combinarse con más categorías léxicas distintas que el que se combina con menos categorías distintas. Las propiedades combinatorias de los predicados afijales del precampo verbal se dan en (6), donde los predicados afijales van seguidos por un paréntesis que contiene las categorías con las que el predicado se combina (Nombre, Adjetivo, Verbo o Adverbio):

(6)

a- (V), æ- (N, V), ar- (V), æt- (N, V, Adj), æfter- (N, V, Adj), be- (N, V), ed- (N, V, Adj), for- $(N, V, A d j)$, for $-(N, V, \operatorname{Adj})$, in- $(N, V, \operatorname{Adj})$, mis- $(N, V)$, ofer- $(N, V, \operatorname{Adj})$, on- (N, V, Adj, Adv), onemn- (V), ongean- (N, V, Adj), od- (V), to- (N, V, Adv), un- (N,

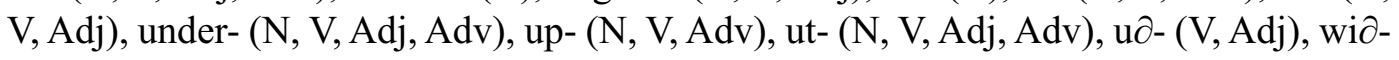
$(\mathrm{N}, \mathrm{V}, \operatorname{Adj}, \mathrm{Adv})$, wider- (N, V, Adv), ymb- (N, V)

Como se muestra en (6), los predicados afijales que se combinan con predicados no afijales de sólo una categoría ( $a$-, ar-, onemn- y $o \partial-$ ) seleccionan predicados verbales. Con la única excepción de $u \partial-$, que se combina con predicados verbales y adjetivales, todos los predicados afijales que se combinan con dos categorías ( $c e-$, be-, mis- y ymb-) seleccionan predicados nominales y verbales. La mayor parte de los predicados afijales se combinan con tres categorías. Éstas son el Nombre, el Verbo y el Adjetivo. Así, los predicados afijales cet-, after, ed-, for-, ford-, in-, ofer-, ongean- y un- se combinan con Nombre, Verbo y Adjetivo, mientras que to-, up- y wider- lo hacen con Nombre, Verbo y Adverbio. Todos los predicados afijales que se combinan con cuatro categorías (on-, under-, ut- y wid-) seleccionan predicados nominales, verbales, adjetivales y adverbiales. No se encuentra evidencia de combinación con la Adposición.

Además de identificar los predicados afijales on-, under-, ut- y wid- como los más productivos cualitativamente desde el punto de vista de la distribución, el análisis ofrecido en (6) nos permite extraer dos conclusiones importantes respecto a la derivación léxica anglosajona. Primero, todos los predicados afijales se combinan con la categoría verbo mientras que ningún predicado afijal de los identificados hasta ahora se combina con la categoría Adposición. Y, segundo, aún con un análisis cuantitativo parcial, ya es posible identificar 
patrones de convergencia entre el análisis cuantitativo y el cualitativo de la productividad de los afijos. Seguramente no es casualidad que el predicado afijal -on sea muy productivo tanto cualitativa como cuantitativamente. El análisis cualitativo desde el punto de vista del comportamiento de los predicados confirmará esta conclusión.

Respecto al comportamiento, entendemos que es más productivo un predicado afijal capaz de cambiar la categoría léxica del predicado fuente de una cadena derivativa que aquel que no lo es. Los predicados afijales que producen recategorización son minoría (González Torres y Martín Arista 2004), como se ilustra en (7). Y de éstos sólo uno muestra dos patrones de recategorización, wider-, dados en (7.g) y (7.h):

a.

$$
\begin{aligned}
& \text { bi }+\mathrm{V}=\text { Adj } \\
& \text { sacan "struggle": biscec "disputed" } \\
& \text { for + N = Adj } \\
& \text { ciele "chill": forcilled "chilled" }
\end{aligned}
$$

b. $\quad$ for $+\mathrm{N}=$ Adj

c. $\quad$ on $+\mathrm{Adj}=\mathrm{Adp}$

middan "middle": onmiddan "at the middle of"

d. $\quad$ ongean $+\mathrm{N}=\mathrm{Adj}$

winnend "fighter": ongeanwinnende "resisting"

e. $\quad$ to $+\mathrm{Adv}=\mathrm{Adp}$

foran "before": toforan "before"

f. $\quad$ un $+\mathrm{N}=$ Adj

ceap "payment": unceap "gratuitous"

g. $\quad$ wider $+\mathrm{N}=\mathrm{Adj}$

scecc "contest": widerscecc "unfavourable"

h. $\quad$ wider $+A d v=A d j$

dune "down": widerdune "steep"

\section{CONCLUSIONES}

El análisis cualitativo de la productividad desde el punto de vista del comportamiento es coincidente con el análisis cuantitativo de la productividad de for-, on- y to-, que se confirman como predicados afijales productivos a falta de llevar a cabo un análisis completo y periodificado. En este artículo también hemos revisado brevemente el estado de la cuestión de la asociación en el léxico anglosajón y hemos propuesto pautas de análisis de la productividad léxica en una lengua de la que sólo contamos con evidencia indirecta (textual). Así, hemos desvinculado la productividad de la frecuencia textual al distinguir la productividad cuantitativa de la cualitativa. En la parte cuantitativa, además de considerar productivos a los predicados afijales de precampo $a-, c_{-}$, be-, for-, ofer-, on- y to-, hemos identificado hapax legomena absolutos y relativos. En cuanto a la productividad cualitativa, hemos demostrado que el análisis del comportamiento y de la distribución tiende a ser coincidente, a lo que se puede concluir que los predicados afijales for-, on- y to- eran productivos en inglés antiguo. 


\section{REFERENCIAS BIBLIOGRÁFICAS}

Aronoff, M. y F. Anshen. 1998. "Morphology and the Lexicon: Lexicalization and Productivity”. The Handbook of Morphology. Eds. A. SPEncer y A. Zwicky. Oxford: Blackwell. 237-247.

Bauer, L. 1983. English Word Formation. Cambridge: Cambridge University Press.

Baugh, A. y T. Cable. 1981 (1957). A History of the English Language. London: Routledge.

Beard, R. 1998. "Derivation". The Handbook of Morphology. Eds. A. Spencer y A. ZwICKY. Oxford: Blackwell. 44-64.

Bosworth, J. y T.N. Toller. 1973 (1898). An Anglo-Saxon Dictionary. Oxford: Oxford University Press.

Diк, S. 1986. "On the notion 'functional explanation"”. Belgian Journal of Linguistics, 1: 11-52.

GonzÁlez Torres, E. y J. Martín Arista. 2004. "Recategorisation and recursion in the derivation of verbal predicates". Comunicación presentada en el XVIII Congreso Internacional de AEDEAN. Valencia, Diciembre 2004.

HiltunEn, R. 1983. The Decline of the Prefixes and the Beginning of the English Phrasal Verb. Turku: Turun Yliopisto.

Katamba, F. 1993. Morphology. London: McMillan.

Kastovsky, D. 1992. "Semantics and vocabulary". The Cambridge History of the English Language. Vol. I: The Beginning to 1066. Ed. R. M. Hogg. Cambridge: Cambridge University Press. 290-408.

Lass, R. y J. Anderson. 1975. Old English Phonology. Cambridge: Cambridge University Press.

Lieber, R. 1992. Deconstructing Morphology. Word Formation in Syntactic Theory. Chicago: The University of Chicago Press.

Plag, I. 1999. Morphological Productivity. Structural Constraints in English Derivation. Berlin: Mouton de Gruyter.

Romano Mozo, M. 2001. "El léxico medieval inglés: características y evolución”. Lingüistica histórica inglesa. Eds. I. DE LA CRUZ y J. Martín ARISTA. Barcelona: Ariel. 378-446.

SPEnCER, A. 1991. Morphological Theory. Oxford: Blackwell.

Stump. G. 2001. Inflectional Morphology. Cambridge: Cambridge University Press. 